\title{
PERIODIC SOLUTIONS FOR A KIND OF $p$-LAPLACIAN HAMILTONIAN SYSTEMS
}

\author{
Li Zhang and Weigao Ge
}

\begin{abstract}
In this paper, the existence of periodic solutions is obtained for a kind of $p$-Laplacian systems by the minimax methods in critical point theory. Moreover, the existence of infinite periodic solutions is also obtained.
\end{abstract}

\section{Introduction}

This paper is concerned with the existence of periodic solutions for the following system

$$
\left\{\begin{aligned}
\frac{d}{d t}\left(\phi_{p}(\dot{u}(t))\right) & =\nabla F(t, u(t)), \text { a.e. } t \in[0, T], \\
u(0)-u(T) & =\dot{u}(0)-\dot{u}(T)=0
\end{aligned}\right.
$$

where $p>1, \phi_{p}(u)=|u|^{p-2} u=\left(\sqrt{\sum_{i=1}^{N} u_{i}^{2}}\right)^{p-2} \cdot\left(u_{1}, u_{2}, \ldots, u_{N}\right), T>0$ and $F:[0, T] \times \mathbb{R}^{N} \rightarrow \mathbb{R}$ satisfies the following assumption:

(A) $F(t, u)$ is measurable in $t$ for each $u \in \mathbb{R}^{N}$ and continuously differentiable in $u$ for a.e. $t \in[0, T]$, and there exist $a \in C\left(\mathbb{R}^{+}, \mathbb{R}^{+}\right), b \in L^{1}\left(0, T ; \mathbb{R}^{+}\right)$such that

$$
|F(t, u)| \leq a(|u|) b(t),|\nabla F(t, u)| \leq a(|u|) b(t)
$$

for all $u \in \mathbb{R}^{N}$ and a.e. $t \in[0, T]$.

When $p=2$, problem (1.1) becomes the second order Hamiltonian systems

$$
\left\{\begin{aligned}
\ddot{u}(t) & =\nabla F(t, u(t)), \text { a.e. } t \in[0, T], \\
u(0)-u(T) & =\dot{u}(0)-\dot{u}(T)=0 .
\end{aligned}\right.
$$

Many existence results for (1.2) are obtained by using variational methods, such as $[2,4,5,6,7]$ and the references therein. Tang [4] gets some existence

Received November 2, 2008.

2000 Mathematics Subject Classification. 34K13, 35A15.

Key words and phrases. periodic solution, $p$-Laplacian system, PS-condition, saddle point theorem.

Supported by the National Natural Science Foundation of China(No. 10671012) and the Doctoral Program Foundation of the Education Ministry of China(20050007011). 
results of (1.2) when $F(t, u)=F_{1}(t, u)+F_{2}(t, u)$ and $\int_{0}^{T} F(t, u) d t \rightarrow+\infty$ as $|u| \rightarrow \infty, F(t, \cdot)$ is subadditive for a.e. $t \in[0, T]$ and there exists $g \in L^{1}[0, T]$ such that $\left|\nabla F_{2}(t, u)\right| \leq g(t)$ for all $u \in \mathbb{R}^{N}$ and a.e. $t \in[0, T]$. Moreover, by the least action principle, $\mathrm{Wu}$ and Tang [6] obtain some existence results of (1.2) while $F_{1}(t, \cdot)$ is $(\lambda, \mu)$-subconvex and $\nabla F_{2}(t, u) \leq f(t)|u|^{\alpha}+g(t)$ for some $0 \leq \alpha<1, f, g \in L^{1}\left(0, T ; \mathbb{R}^{+}\right)$.

However, while $F(t, u) \rightarrow-\infty$ as $|u| \rightarrow+\infty$ uniformly for a.e. $t \in[0, T]$, we do not know whether the question is a positive answer. Moreover, for the general case $p>1$, some papers calling it vector $p$-Laplacian, there are not so many results. Differential equations with $p$-Laplacian have many important applications such as non-Newtonian fluid theory and the turbulent flow of the gas in porous medium. Motivated by the work mentioned above, in this paper, we consider the existence of periodic solutions for problem (1.1).

\section{Preliminary}

The Sobolev space $W_{T}^{1, p}$ is defined by

$$
\begin{gathered}
W_{T}^{1, p}=\left\{u:[0, T] \rightarrow \mathbb{R}^{N} \mid u \text { is absolutely continuous, } u(0)=u(T),\right. \\
\text { and } \left.\dot{u} \in L^{p}\left(0, T ; \mathbb{R}^{N}\right)\right\}
\end{gathered}
$$

and is endowed with the norm

$$
\|u\|=\left(\int_{0}^{T}|u(t)|^{p} d t+\int_{0}^{T}|\dot{u}(t)|^{p} d t\right)^{\frac{1}{p}} .
$$

It follows from [2] that $W_{T}^{1, p}$ is a reflexive Banach space. Moreover, $W_{T}^{1, p}$ is a closed subspace of $W_{T}^{1, p}$ under (2.2). From [1, Theorem 1.21] and [1, Theorem 3.5], we obtain $W_{T}^{1, p}$ is uniformly convex.

Define a functional $\varphi$ on $W_{T}^{1, p}$ by

$$
\varphi(u)=\frac{1}{p} \int_{0}^{T}|\dot{u}(t)|^{p} d t+\int_{0}^{T} F(t, u(t)) d t, u \in W_{T}^{1, p} .
$$

It follows from assumption $(A)$ that the functional $\varphi$ is continuously differentiable on $W_{T}^{1, p}$. Moreover,

$$
\left\langle\varphi^{\prime}(u), v\right\rangle=\int_{0}^{T}\left[\left(|\dot{u}(t)|^{p-2} \dot{u}(t), \dot{v}(t)\right)+(\nabla F(t, u(t)), v(t))\right] d t
$$

for all $u, v \in W_{T}^{1, p}$. It is well known that the solutions of problem (1.1) correspond to the critical points of $\varphi$.

The following lemmas are basic in our paper. 
Lemma $2.1([2])$. Set $\|u\|_{\infty}=\max _{t \in[0, T]}|u(t)|,\|u\|_{L^{p}}=\left(\int_{0}^{T}|u(t)|^{p} d t\right)^{\frac{1}{p}}$. If $u \in W_{T}^{1, p}$ and $\int_{0}^{T} u(t) d t=0$, then

$$
\|u\|_{\infty} \leq T^{\frac{1}{q}}\|\dot{u}\|_{L^{p}},\left(\frac{1}{p}+\frac{1}{q}=1\right) .
$$

Moreover, we have

$$
\|u\|_{L^{p}} \leq T\|\dot{u}\|_{L^{p}}
$$

Lemma $2.2([5])$. Suppose $F(t, u)$ satisfies the assumption $(A)$ and $E$ is a measurable subset of $[0, T]$. Assume

$$
F(t, u) \rightarrow-\infty,|u| \rightarrow \infty
$$

for a.e. $t \in E$. Then, for every $\delta>0$, there exists a subset $E_{\delta}$ of $E$ with $\operatorname{meas}\left(E \backslash E_{\delta}\right)<\delta$ such that

$$
F(t, u) \rightarrow-\infty,|u| \rightarrow \infty
$$

uniformly for all $t \in E_{\delta}$.

Theorem 2.1 ([2, The Saddle Point Theorem]). Let $X$ be a Banach space and let $\varphi \in C^{1}(X, R)$. Assume that $X$ splits into a direct sum of closed subspaces $X=X^{-} \oplus X^{+}$with

$$
\operatorname{dim} X^{-}<\infty
$$

and

$$
\sup _{S_{R}^{-}} \varphi<\inf _{X^{+}} \varphi
$$

where $S_{R}^{-}=\left\{u \in X^{-}:|u|=R\right\}$. Let

$$
\begin{gathered}
B_{R}^{-}=\left\{u \in X^{-}:|u| \leq R\right\}, \\
M=\left\{g \in C\left(B_{R}^{-}, X\right): g(s)=s \text { if } s \in S_{R}^{-}\right\}
\end{gathered}
$$

and

$$
c=\inf _{g \in M} \max _{s \in B_{R}^{-}} \varphi(g(s)) .
$$

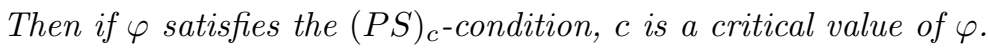

Theorem $2.2([3])$. Assume $E$ is a real Banach space, $I \in C^{1}(E, R)$ is an even functional satisfying $P S$-condition and $I(0)=0$. If $E=V \oplus X, \operatorname{dim} V<\infty$ and $I$ satisfies

$\left(A_{1}\right)$ there exist constants $\rho, \alpha>0$ such that $\left.I\right|_{\partial B_{\rho} \cap X} \geq \alpha$;

$\left(A_{2}\right)$ for $\forall V_{1} \subset E$ with $\operatorname{dim} V_{1}<\infty$, the set $\left\{x \in V_{1}: I(x) \geq 0\right\}$ is bounded, then $I$ has an unbounded critical value sequence. 


\section{Main results}

Suppose $F(t, u)=G(u)+H(t, u)$ and

$\left(B_{1}\right)$ there exist $f, g \in L^{1}\left(0, T ; R^{+}\right)$such that

$$
|\nabla H(t, u)| \leq f(t)|u|^{\alpha}+g(t)
$$

holds for all $u \in \mathbb{R}^{N}$ and a.e. $t \in[0, T]$. Here $\alpha$ satisfies

$$
0 \leq \alpha<1, \text { if } p \geq q \text { and } 0 \leq \alpha<\frac{p}{q} \text {, if } p<q
$$

$\left(B_{2}\right)$ there exists a constant $r<\frac{1-T^{\frac{p}{q}}}{T^{p}}$ such that

$$
(\nabla G(u)-\nabla G(v), u-v) \geq-r|u-v|^{p}, u, v \in \mathbb{R}^{N}
$$

$\left(B_{3}\right)$ there exists a subset $E$ of $[0, T]$ with meas $(E)>0$ such that

$$
|u|^{-\max \{p, q\} \alpha} F(t, u) \rightarrow-\infty \text { as }|u| \rightarrow \infty
$$

for a.e. $t \in E$;

$\left(B_{4}\right)$ there exist $c(t) \in L^{1}\left(0, T ; \mathbb{R}^{+}\right), d(t) \in L^{1}(0, T), \gamma \geq 0$ such that

$$
F(t, u) \leq c(t)|u|^{-\gamma}+d(t)
$$

for $u \in \mathbb{R}^{N}, u \neq \theta$, and a.e. $t \in[0, T]$.

In the following, for $u \in W_{T}^{1, p}$, let $\bar{u}=\frac{1}{T} \int_{0}^{T} u(t) d t$ and $\tilde{u}=u-\bar{u}$.

Lemma 3.1. Suppose $F(t, u)$ satisfy $(A)$ and $\left(B_{1}\right)-\left(B_{4}\right)$. Then $\varphi$ satisfies the PS-condition, that is, $\left(u_{n}\right)_{n \in \mathbb{N}}$ has a convergent subsequence whenever it satisfies $\varphi^{\prime}\left(u_{n}\right) \rightarrow 0$ as $n \rightarrow \infty$ and $\varphi\left(u_{n}\right)$ is bounded.

Proof. By (2.6), we have

$$
\begin{aligned}
\left(\int_{0}^{T}|\dot{u}(t)|^{p} d t\right)^{\frac{1}{p}} & =\left(\int_{0}^{T}|\dot{\tilde{u}}(t)|^{p} d t\right)^{\frac{1}{p}} \leq\|\tilde{u}\| \\
& =\left(\int_{0}^{T}|\tilde{u}(t)|^{p} d t+\int_{0}^{T}|\dot{\tilde{u}}(t)|^{p} d t\right)^{\frac{1}{p}} \\
& \leq\|\tilde{u}\|_{L^{p}}+\|\dot{\tilde{u}}\|_{L^{p}} \leq(T+1)\|\dot{\tilde{u}}\|_{L^{p}} .
\end{aligned}
$$

By $\left(B_{2}\right)$ and $(2.6)$, we obtain

$$
\begin{aligned}
\int_{0}^{T}(\nabla G(u(t)), \tilde{u}(t)) d t & =\int_{0}^{T}(\nabla G(u(t))-\nabla G(\bar{u}(t)), \tilde{u}(t)) d t \\
& =\int_{0}^{T}(\nabla G(u(t))-\nabla G(\bar{u}(t)), u(t)-\bar{u}(t)) d t \\
& \geq-r \int_{0}^{T}|u-\bar{u}|^{p} d t \\
& =-r\|\tilde{u}\|_{L^{p}}^{p} \geq-r T^{p}\|\dot{u}\|_{L^{p}}^{p}, \forall u \in W_{T}^{1, p} .
\end{aligned}
$$


Suppose $\left(u_{n}\right)_{n \in \mathbb{N}}$ is a sequence satisfying $\varphi^{\prime}\left(u_{n}\right) \rightarrow 0$ as $n \rightarrow \infty$ and $\varphi\left(u_{n}\right)$ is bounded. In order to prove

$$
\left|u_{n}(t)\right| \geq C_{0}\left|\bar{u}_{n}\right|-C_{0}^{\prime}, \forall t \in[0, T]
$$

holds for some positive constants $C_{0}, C_{0}^{\prime}$, there are two cases.

Case 1. $p \geq q$.

From $\left(B_{1}\right)$ and $(2.5)$,

$$
\begin{aligned}
& \left|\int_{0}^{T}(\nabla H(t, u(t)), \tilde{u}(t)) d t\right| \\
\leq & \int_{0}^{T} f(t)|u(t)|^{\alpha}|\tilde{u}(t)| d t+\int_{0}^{T} g(t)|\tilde{u}(t)| d t \\
\leq & \left(|\bar{u}|^{\alpha}+\|\tilde{u}(t)\|_{\infty}^{\alpha}\right)\|\tilde{u}(t)\|_{\infty} \int_{0}^{T} f(t) d t+\|\tilde{u}(t)\|_{\infty} \int_{0}^{T} g(t) d t \\
= & |\bar{u}|^{\alpha}\|\tilde{u}(t)\|_{\infty} \int_{0}^{T} f(t) d t+\|\tilde{u}(t)\|_{\infty}^{\alpha+1} \int_{0}^{T} f(t) d t+\|\tilde{u}(t)\|_{\infty} \int_{0}^{T} g(t) d t \\
\leq & \|\tilde{u}(t)\|_{\infty}^{q}+|\bar{u}|^{p \alpha}\left(\int_{0}^{T} f(t) d t\right)^{p}+\|\tilde{u}(t)\|_{\infty}^{\alpha+1} \int_{0}^{T} f(t) d t+\|\tilde{u}(t)\|_{\infty} \int_{0}^{T} g(t) d t \\
\leq & T\|\dot{u}\|_{L^{p}}^{q}+C_{1}|\bar{u}|^{p \alpha}+C_{2}\|\dot{u}\|_{L^{p}}^{\alpha+1}+C_{3}\|\dot{u}\|_{L^{p}}
\end{aligned}
$$

holds for all $u \in W_{T}^{1, p}$ and some positive constants $C_{1}, C_{2}$ and $C_{3}$. Hence, for large $n$, one has

$$
\begin{aligned}
\left\|\tilde{u}_{n}\right\| & \geq\left|\left\langle\varphi^{\prime}\left(u_{n}\right), \tilde{u}_{n}\right\rangle\right|=\left.\left|\int_{0}^{T}\right| \dot{u}_{n}(t)\right|^{p} d t+\int_{0}^{T}\left(\nabla F\left(t, u_{n}(t)\right), \tilde{u}_{n}(t)\right) d t \mid \\
& =\left.\left|\int_{0}^{T}\right| \dot{u}_{n}(t)\right|^{p} d t+\int_{0}^{T}\left(\nabla G\left(u_{n}(t)\right), \tilde{u}_{n}(t)\right) d t+\int_{0}^{T}\left(\nabla H\left(t, u_{n}(t)\right), \tilde{u}_{n}(t)\right) d t \mid \\
& \geq\left\|\dot{u}_{n}\right\|_{L^{p}}^{p}-r T^{p}\left\|\left.\dot{u}_{n}\right|_{L^{p}} ^{p}-T\right\| \dot{u}_{n}\left\|_{L^{p}}^{q}-C_{1}\left|\bar{u}_{n}\right|^{p \alpha}-C_{2}\right\| \dot{u}_{n}\left\|_{L^{p}}^{\alpha+1}-C_{3}\right\| \dot{u}_{n} \|_{L^{p}} .
\end{aligned}
$$

Then, by (3.1) and (3.4)

$C_{1}\left|\bar{u}_{n}\right|^{p \alpha} \geq\left(1-r T^{p}\right)|| \dot{u}_{n}\left\|_{L^{p}}^{p}-\left.T|| \dot{u}_{n}\right|_{L^{p}} ^{q}-\left.C_{2}|| \dot{u}_{n}\right|_{L^{p}} ^{\alpha+1}-\left(1+T+C_{3}\right)|| \dot{u}_{n}\right\|_{L^{p}}$.

Obviously, $p \geq 2$ and $\alpha<1$. Moreover, from $\left(B_{2}\right)$ one has $1-r T^{p}>0$ for $p>q$, and $1-r T^{p}-T>0$ for $p=q$. Hence,

$$
C_{4}\left|\bar{u}_{n}\right|^{\alpha} \geq\left\|\dot{u}_{n}\right\|_{L^{p}}-C_{5}
$$

holds for some constants $C_{4}, C_{5}$ and large $n$. This implies that

$$
\left\|\tilde{u}_{n}\right\|_{\infty} \leq C_{6}\left(\left|\bar{u}_{n}\right|^{\alpha}+1\right)
$$


for some constants $C_{6} \neq 1$ and large $n$. Then one has

$$
\begin{aligned}
\left|u_{n}(t)\right| \geq\left|\bar{u}_{n}\right|-\left|\tilde{u}_{n}(t)\right| & \geq\left|\bar{u}_{n}\right|-\left.|| \tilde{u}_{n}(t)\right|_{\infty} \\
& \geq\left|\bar{u}_{n}\right|-C_{6}\left(\left|\bar{u}_{n}\right|^{\alpha}+1\right) \\
& =\left|\bar{u}_{n}\right|\left[1-C_{6}\left(\left|\bar{u}_{n}\right|^{\alpha-1}+\left|\bar{u}_{n}\right|^{-1}\right)\right]
\end{aligned}
$$

for large $n$ and $t \in[0, T]$. If $\left(\left|\bar{u}_{n}\right|\right)_{n \in \mathbb{N}}$ is bounded, we can easily obtain (3.2). If $\left(\left|\bar{u}_{n}\right|\right)_{n \in \mathbb{N}}$ is unbounded, without loss of generality, we may suppose $\left|\bar{u}_{n}\right| \rightarrow \infty$ as $n \rightarrow \infty$, then, for sufficiently small $\varepsilon>0$ and large $n,\left|\bar{u}_{n}\right|^{\alpha-1}<\varepsilon,\left|\bar{u}_{n}\right|^{-1}<\varepsilon$. Hence, (3.2) holds.

Case 2. $p<q$.

Similarly, we have

$$
\begin{aligned}
& \left|\int_{0}^{T}(\nabla H(t, u(t)), \tilde{u}(t)) d t\right| \\
\leq & \int_{0}^{T} f(t)|u(t)|^{\alpha}|\tilde{u}(t)| d t+\int_{0}^{T} g(t)|\tilde{u}(t)| d t \\
\leq & \|\tilde{u}(t)\|_{\infty}^{p}+|\bar{u}|^{q \alpha}\left(\int_{0}^{T} f(t) d t\right)^{q}+\|\tilde{u}(t)\|_{\infty}^{\alpha+1} \int_{0}^{T} f(t) d t+\|\tilde{u}(t)\|_{\infty} \int_{0}^{T} g(t) d t
\end{aligned}
$$

$\leq T^{\frac{p}{q}}\|\dot{u}\|_{L^{p}}^{p}+C_{1}^{\prime}|\bar{u}|^{q \alpha}+C_{2}\|\dot{u}\|_{L^{p}}^{\alpha+1}+C_{3}\|\dot{u}\|_{L^{p}}$

holds for all $u \in W_{T}^{1, p}$ and some positive constant $C_{1}^{\prime}$. Hence, for large $n$, one has

$$
\begin{aligned}
\left\|\tilde{u}_{n}\right\| & \geq\left|\left\langle\varphi^{\prime}\left(u_{n}\right), \tilde{u}_{n}\right\rangle\right| \\
& \geq\left(1-r T^{p}-T^{\frac{p}{q}}\right)|| \dot{u}_{n}\left\|_{L^{p}}^{p}-C_{1}^{\prime}\left|\bar{u}_{n}\right|^{q \alpha}-C_{2}\right\| \dot{u}_{n}\left\|_{L^{p}}^{\alpha+1}-C_{3}\right\| \dot{u}_{n} \|_{L^{p}} .
\end{aligned}
$$

Then,

$$
\begin{aligned}
C_{1}^{\prime}\left|\bar{u}_{n}\right|^{q \alpha} & \geq\left(1-r T^{p}-T^{\frac{p}{q}}\right)\left\|\dot{u}_{n}\right\|_{L^{p}}^{p}-C_{2}\left\|\dot{u}_{n}\right\|_{L^{p}}^{\alpha+1}-\left(1+T+C_{3}\right)\left\|\dot{u}_{n}\right\|_{L^{p}} \\
& =\left\|\dot{u}_{n}\right\|_{L^{p}}^{p}\left[\left(1-r T^{p}-T^{\frac{p}{q}}\right)-C_{2}\left\|\dot{u}_{n}\right\|_{L^{p}}^{\alpha+1-p}-\left(1+T+C_{3}\right)\left\|\dot{u}_{n}\right\|_{L^{p}}^{1-p}\right] .
\end{aligned}
$$

From $\left(B_{1}\right),\left(B_{2}\right)$, one has $\alpha+1<\frac{p}{q}+1=p$ and $1-r T^{p}-T^{\frac{p}{q}}>0$. Hence, with the similar discussion above,

$$
C_{4}^{\prime}\left|\bar{u}_{n}\right|^{q \alpha} \geq \|\left.\dot{u}_{n}\right|_{L^{p}} ^{p}-C_{5}^{\prime}
$$

holds for some constants $C_{4}^{\prime}, C_{5}^{\prime}$ and large $n$, that is,

$$
\left\|\tilde{u}_{n}\right\|_{\infty}^{\frac{p}{q}} \leq C_{6}^{\prime}\left(\left|\bar{u}_{n}\right|^{\alpha}+1\right)
$$

for some constants $C_{6}^{\prime} \neq 1$ and large $n$. Then,

$$
\left|u_{n}(t)\right|^{\frac{p}{q}} \geq\left|\bar{u}_{n}\right|^{\frac{p}{q}}-\|\left.\tilde{u}_{n}(t)\right|_{\infty} ^{\frac{p}{q}} \geq\left|\bar{u}_{n}\right|^{\frac{p}{q}}-C_{6}^{\prime}\left(\left|\bar{u}_{n}\right|^{\alpha}+1\right)
$$

for large $n$ and $t \in[0, T]$. 
Then from (3.7) and (3.10), whenever $p \geq q$ or $p<q$, (3.2) holds for some constants $C_{0}, C_{0}^{\prime}$ and large $n$. If $\left(\left|\bar{u}_{n}\right|\right)_{n \in \mathbb{N}}$ is unbounded, we may assume that, going to a subsequence if necessary,

$$
\left|\bar{u}_{n}\right| \rightarrow \infty \text { as } n \rightarrow \infty \text {. }
$$

Set $\delta=\frac{m(E)}{2}$. It follows from Lemma 2.2 and $\left(B_{3}\right)$ that there exists a subset $E_{\delta}$ of $E$ with meas $\left(E \backslash E_{\delta}\right)<\delta$ such that

$$
|u|^{-\max \{p, q\} \alpha} F(t, u) \rightarrow-\infty \quad \text { as }|u| \rightarrow \infty
$$

uniformly for all $t \in E_{\delta}$, which implies that

$$
m\left(E_{\delta}\right)=m(E)-m\left(E \backslash E_{\delta}\right)>\delta>0 .
$$

Moreover, for every $N>0$, there exists $M \geq 1$ such that

$$
|u|^{-\max \{p, q\} \alpha} F(t, u) \leq-N
$$

for all $|u| \geq M$ and $t \in E_{\delta}$. By (3.2) and (3.11), one has

$$
\left|u_{n}(t)\right| \geq M
$$

for large $n$ and every $t \in[0, T]$. Then

$$
\begin{aligned}
\varphi\left(u_{n}\right)= & \frac{1}{p} \int_{0}^{T}\left|\dot{u}_{n}(t)\right|^{p} d t+\int_{0}^{T} F\left(t, u_{n}(t)\right) d t \\
= & \frac{1}{p} \|\left.\dot{u}_{n}(t)\right|_{L^{p}} ^{p}+\int_{E_{\delta}} F\left(t, u_{n}(t)\right) d t+\int_{[0, T] \backslash E_{\delta}} F\left(t, u_{n}(t)\right) d t \\
\leq & \frac{1}{p} \|\left.\dot{u}_{n}(t)\right|_{L^{p}} ^{p}-N \int_{E_{\delta}}\left|u_{n}(t)\right|^{\max \{p, q\} \alpha} d t+\int_{[0, T] \backslash E_{\delta}} c(t)\left|u_{n}\right|^{-\gamma} d t \\
& +\int_{[0, T] \backslash E_{\delta}} d(t) d t \\
\leq & \left.\frac{1}{p}|| \dot{u}_{n}(t)\right|_{L^{p}} ^{p}-2^{-\max \{p, q\} \alpha} N \delta C_{0}^{\max \{p, q\} \alpha}\left|\bar{u}_{n}\right|^{\max \{p, q\} \alpha} \\
& +N \delta C_{0}^{\prime \max \{p, q\} \alpha}+M^{-\gamma} \int_{[0, T] \backslash E_{\delta}} c(t) d t+\int_{[0, T] \backslash E_{\delta}} d(t) d t \\
\leq & \left\{\begin{array}{l}
\frac{1}{p}\left(C_{4}\left|\bar{u}_{n}\right|^{\alpha}+C_{5}\right)^{p}-\left(2^{-p \alpha} C_{0}^{p \alpha}\left|\bar{u}_{n}\right|^{p \alpha}-C_{0}^{\prime p \alpha}\right) N \delta \\
+M^{-\gamma} \int_{[0, T] \backslash E_{\delta}} c(t) d t+\int_{[0, T] \backslash E_{\delta}} d(t) d t, \text { if } p \geq q, \\
\frac{1}{p}\left(\left.C_{4}^{\prime}\left|\bar{u}_{n}\right|\right|^{q \alpha}+C_{5}^{\prime}\right)-\left(2^{-q \alpha} C_{0}^{q \alpha}\left|\bar{u}_{n}\right|^{q \alpha}-C_{0}^{\prime q \alpha}\right) N \delta \\
+M^{-\gamma} \int_{[0, T] \backslash E_{\delta}} c(t) d t+\int_{[0, T] \backslash E_{\delta}} d(t) d t, \text { if } p<q
\end{array}\right.
\end{aligned}
$$

for large $n$. Hence, we have

$\limsup _{n \rightarrow \infty}\left|\bar{u}_{n}\right|^{-\max \{p, q\} \alpha} \varphi\left(u_{n}\right) \leq-2^{-\max \{p, q\} \alpha} C_{0}^{\max \{p, q\} \alpha} \delta N+\frac{1}{p} \max \left\{C_{4}^{p}, C_{4}^{\prime p}\right\}$.

By the arbitrariness of $N>0$, one has

$$
\limsup _{n \rightarrow \infty}\left|\bar{u}_{n}\right|^{-\max \{p, q\} \alpha} \varphi\left(u_{n}\right)=-\infty
$$


which contradicts with the boundedness of $\varphi\left(u_{n}\right)$. Therefore, $\left(\left|\bar{u}_{n}\right|\right)_{n \in \mathbb{N}}$ is bounded. Furthermore, by (3.5), (3.8), and

$$
\left\|u_{n}\right\| \leq\left\|\bar{u}_{n}\right\|+(T+1)\left\|\dot{\tilde{u}}_{n}\right\|_{L^{p}},
$$

one has $\left(u_{n}\right)_{n \in \mathbb{N}}$ is bounded in $W_{T}^{1, p}$. Going if necessary to a subsequence, we can assume that $u_{n} \rightarrow u$ in $W_{T}^{1, p}$ and $u_{n} \rightarrow u$ in $C\left([0, T], R^{N}\right)$. Hence,

$$
\begin{aligned}
& \left\langle\varphi^{\prime}\left(u_{n}\right), u_{n}-u\right\rangle \\
= & \int_{0}^{T}\left[\left(\left|\dot{u}_{n}(t)\right|^{p-2} \dot{u}_{n}(t), \dot{u}_{n}(t)-\dot{u}(t)\right)+\left(\nabla F\left(t, u_{n}(t)\right), u_{n}(t)-u(t)\right)\right] d t \rightarrow 0 \\
& \quad \operatorname{as~} n \rightarrow \infty,
\end{aligned}
$$

and $\left(u_{n}\right)_{n \in \mathbb{N}}$ is bounded in $C\left([0, T], R^{N}\right)$. Therefore

$$
\int_{0}^{T}\left(\left|u_{n}\right|^{p-2} u_{n}, u_{n}-u\right) d t \rightarrow 0 \text { as } n \rightarrow \infty .
$$

By assumption $(A)$,

$$
\begin{aligned}
\left|\int_{0}^{T}\left(\nabla F\left(t, u_{n}(t)\right), u_{n}(t)-u(t)\right) d t\right| & \leq \int_{0}^{T}\left|\nabla F\left(t, u_{n}(t)\right)\right| \cdot\left|u_{n}(t)-u(t)\right| d t \\
& \leq C_{7}|| b||_{L^{1}}\left\|u_{n}-u\right\|_{\infty}
\end{aligned}
$$

holds for some positive constant $C_{7}$. Then

$$
\int_{0}^{T}\left(\nabla F\left(t, u_{n}(t)\right), u_{n}(t)-u(t)\right) d t \rightarrow 0 \text { as } n \rightarrow \infty .
$$

From (3.14) and (3.16), we obtain

$$
\int_{0}^{T}\left(\left|\dot{u}_{n}(t)\right|^{p-2} \dot{u}_{n}(t), \dot{u}_{n}(t)-\dot{u}(t)\right) d t \rightarrow 0 \quad \text { as } n \rightarrow \infty .
$$

Set $\psi(u)=\frac{1}{p}\|u\|^{p}=\frac{1}{p}\left(\int_{0}^{T}|u|^{p} d t+\int_{0}^{T}|\dot{u}|^{p} d t\right)$. By (3.15) and (3.17), we have

$$
\begin{aligned}
& \left\langle\psi^{\prime}\left(u_{n}\right), u_{n}-u\right\rangle \\
= & \int_{0}^{T}\left(\left|u_{n}\right|^{p-2} u_{n}, u_{n}-u\right) d t+\int_{0}^{T}\left(\left|\dot{u}_{n}\right|^{p-2} \dot{u}_{n}, \dot{u}_{n}-\dot{u}\right) d t \rightarrow 0 \text { as } n \rightarrow \infty .
\end{aligned}
$$

Using the Hölder's inequality, we have

$$
\begin{aligned}
& \left\langle\psi^{\prime}\left(u_{n}\right)-\psi^{\prime}(u), u_{n}-u\right\rangle \\
= & \int_{0}^{T}\left(\left|u_{n}\right|^{p-2} u_{n}, u_{n}-u\right) d t+\int_{0}^{T}\left(\left|\dot{u}_{n}\right|^{p-2} \dot{u}_{n}, \dot{u}_{n}-\dot{u}\right) d t
\end{aligned}
$$




$$
\begin{aligned}
& -\int_{0}^{T}\left(|u|^{p-2} u, u_{n}-u\right) d t-\int_{0}^{T}\left(|\dot{u}|^{p-2} \dot{u}, \dot{u}_{n}-\dot{u}\right) d t \\
\geq & \int_{0}^{T}\left|u_{n}\right|^{p} d t+\int_{0}^{T}\left|\dot{u}_{n}\right|^{p} d t+\int_{0}^{T}|u|^{p} d t+\int_{0}^{T}|\dot{u}|^{p} d t \\
& -\left(\int_{0}^{T}\left|u_{n}\right|^{p} d t\right)^{\frac{1}{q}}\left(\int_{0}^{T}|u|^{p} d t\right)^{\frac{1}{p}}-\left(\int_{0}^{T}\left|\dot{u}_{n}\right|^{p} d t\right)^{\frac{1}{q}}\left(\int_{0}^{T}|\dot{u}|^{p} d t\right)^{\frac{1}{p}} \\
& -\left(\int_{0}^{T}|u|^{p} d t\right)^{\frac{1}{q}}\left(\int_{0}^{T}\left|u_{n}\right|^{p} d t\right)^{\frac{1}{p}}-\left(\int_{0}^{T}|\dot{u}|^{p} d t\right)^{\frac{1}{q}}\left(\int_{0}^{T}\left|\dot{u}_{n}\right|^{p} d t\right)^{\frac{1}{p}} \\
\geq & \left\|u_{n}||^{p}+|| u\right\|^{p}-\left[\int_{0}^{T}\left|u_{n}\right|^{p} d t+\int_{0}^{T}\left|\dot{u}_{n}\right|^{p} d t\right]^{\frac{1}{q}} \cdot\left[\int_{0}^{T}|u|^{p} d t+\int_{0}^{T}|\dot{u}|^{p} d t\right]^{\frac{1}{p}} \\
& -\left[\int_{0}^{T}\left|u_{n}\right|^{p} d t+\int_{0}^{T}\left|\dot{u}_{n}\right|^{p} d t\right]^{\frac{1}{p}} \cdot\left[\int_{0}^{T}|u|^{p} d t+\int_{0}^{T}|\dot{u}|^{p} d t\right]^{\frac{1}{q}} \\
= & \left(\left\|u_{n}\right\|^{p-1}-\|u\|^{p-1}\right)\left(|| u_{n}\|-\| u \|\right) \geq 0 .
\end{aligned}
$$

From (3.18) and (3.19), one has $\left\|u_{n}\right\| \rightarrow\|u\|$. Then, $u_{n} \rightarrow u$ holds in $W_{T}^{1, p}$ by the uniform convexity of $W_{T}^{1, p}$. Therefore, the PS-condition holds.

Theorem 3.1. Assume $F(t, u)$ satisfy $(A)$ and $\left(B_{1}\right)-\left(B_{4}\right)$. Then problem $(1.1)$ has at least one solution in $W_{T}^{1, p}$.

Proof. It remains to show that $\varphi$ satisfies other conditions of the Saddle Point Theorem. Let $\tilde{W}_{T}^{1, p}$ be the subspace of $W_{T}^{1, p}$ given by

$$
\tilde{W}_{T}^{1, p}=\left\{u \in W_{T}^{1, p} \mid \bar{u}=0\right\} .
$$

Then $W_{T}^{1, p}=R^{N} \oplus \tilde{W}_{T}^{1, p}$. By (2.5), one obtains

$$
\begin{aligned}
& \left|\int_{0}^{T}[H(t, u(t))-H(t, 0)] d t\right| \\
= & \left|\int_{0}^{T} \int_{0}^{1}(\nabla H(t, s u(t)), u(t)) d s d t\right| \\
\leq & \int_{0}^{T} \int_{0}^{1} f(t)|s u(t)|^{\alpha}|u(t)| d s d t+\int_{0}^{T} \int_{0}^{1} g(t)|u(t)| d s d t \\
\leq & \int_{0}^{T} f(t)|u(t)|^{\alpha+1} d t+\int_{0}^{T} g(t)|u(t)| d t \\
\leq & \|u(t)\|_{\infty}^{\alpha+1} \int_{0}^{T} f(t) d t+\|u(t)\|_{\infty} \int_{0}^{T} g(t) d t \\
\leq & T^{\frac{\alpha+1}{q}}\|\dot{u}(t)\|_{L^{p}}^{\alpha+1} \int_{0}^{T} f(t) d t+T^{\frac{1}{q}}\|\dot{u}(t)\|_{L^{p}} \int_{0}^{T} g(t) d t
\end{aligned}
$$




$$
=C_{8}\|\dot{u}(t)\|_{L^{p}}^{\alpha+1}+C_{9}\|\dot{u}(t)\|_{L^{p}}
$$

for all $u \in \tilde{W}_{T}^{1, p}$ and some positive constants $C_{8}$ and $C_{9}$. Moreover,

$$
\begin{aligned}
\int_{0}^{T}[G(u(t))-G(0)] d t & =\int_{0}^{T} \int_{0}^{1}(\nabla G(s u(t)), u(t)) d s d t \\
& =\int_{0}^{T} \int_{0}^{1}(\nabla G(s u(t))-\nabla G(0), u(t)) d s d t \\
& =\int_{0}^{T} \int_{0}^{1} \frac{1}{s}(\nabla G(s u(t))-\nabla G(0), s u(t)) d s d t \\
& \geq \int_{0}^{T} \int_{0}^{1} \frac{1}{s}\left(-r|s u(t)|^{p}\right) d s d t \\
& =-r \int_{0}^{T} \int_{0}^{1} s^{p-1}|u(t)|^{p} d s d t \\
& =-\frac{r}{p}\|u\|_{L^{p}}^{p}
\end{aligned}
$$

for all $u \in \tilde{W}_{T}^{1, p}$. Hence

$$
\begin{aligned}
\varphi(u)-\int_{0}^{T} F(t, 0) d t= & \frac{1}{p} \int_{0}^{T}|\dot{u}(t)|^{p} d t+\int_{0}^{T}[F(t, u(t))-F(t, 0)] d t \\
= & \frac{1}{p} \int_{0}^{T}|\dot{u}(t)|^{p} d t+\int_{0}^{T}[G(u(t))-G(0)] d t \\
& +\int_{0}^{T}[H(t, u(t))-H(t, 0)] d t \\
\geq & \frac{1}{p}\|\dot{u}\|_{L^{p}}^{p}-\frac{r}{p}\|u\|_{L^{p}}^{p}-C_{8}\|\dot{u}\|_{L^{p}}^{\alpha+1}-C_{9}\|\dot{u}\|_{L^{p}} \\
\geq & \frac{1-r T^{p}}{p}\|\dot{u}\|_{L^{p}}^{p}-C_{8}\|\dot{u}\|_{L^{p}}^{\alpha+1}-C_{9}\|\dot{u}\|_{L^{p}}
\end{aligned}
$$

Let $\|u\| \rightarrow \infty$ in $\tilde{W}_{T}^{1, p}$. Then $\|\dot{u}\|_{L^{p}} \rightarrow \infty$ or $\|u\|_{L^{p}} \rightarrow \infty$. From (2.6), one has $\|\dot{u}\|_{L^{p}} \rightarrow \infty$ if $\|u\|_{L^{p}} \rightarrow \infty$. Moreover, from $\left(B_{1}\right), \alpha+1<p$, hence, we have $\varphi(u) \rightarrow+\infty$ while $\|u\| \rightarrow \infty$ in $\tilde{W}_{T}^{1, p}$. On the other hand, for $\forall u \in R^{N}$, we have

$$
\begin{aligned}
\varphi(u) & =\int_{0}^{T} F(t, u) d t \\
& \leq \int_{[0, T] \backslash E_{\delta}}\left(c(t)|u|^{-\gamma}+d(t)\right) d t-\int_{E_{\delta}} N|u|^{(p+q) \alpha} d t \\
& \leq M^{-\gamma} \int_{[0, T] \backslash E_{\delta}} c(t) d t+\int_{[0, T] \backslash E_{\delta}} d(t) d t-\operatorname{meas}\left(E_{\delta}\right) N M^{(p+q) \alpha}
\end{aligned}
$$




$$
\leq M^{-\gamma} \int_{[0, T] \backslash E_{\delta}} c(t) d t+\int_{[0, T] \backslash E_{\delta}} d(t) d t-\operatorname{meas}\left(E_{\delta}\right) N, \forall|u| \geq M .
$$

It is easy to obtain that

$$
\varphi(u) \rightarrow-\infty,|u| \rightarrow \infty, u \in \mathbb{R}^{N} .
$$

Then, from the Saddle Point Theorem, we obtain that the problem (1.1) has at least one solution.

Remark 3.1. If $\nabla F(t, \theta) \not \equiv \theta$ for $t \in[0, T]$, the Hamiltonian system (1.1) has non-trivial solutions.

Theorem 3.2. Assume $F(t, u)$ satisfy $(A)$ and $\left(B_{1}\right)-\left(B_{4}\right)$ and the following conditions:

$\left(B_{5}\right) F(t, \theta)=0, F(t,-u)=F(t, u)$;

$\left(B_{6}\right)$ there exists $e(t) \in L^{1}\left(0, T ; R^{+}\right)$and constants $\beta$ satisfying $\beta>p$ and $M^{\prime}>0$ such that $F(t, u) \geq-e(t)|u|^{\beta}$ holds for $u \in \tilde{W}_{T}^{1, p}$ and $\|u\| \leq$ $M^{\prime}$;

$\left(B_{7}\right)$ there exists $l(t)$ satisfying $\int_{0}^{T} l(t) d t>0$ and constants $m>p$ and $M^{\prime \prime}>M^{\prime}$ such that $\int_{0}^{T} F(t, u) d t \leq-\int_{0}^{T} l(t) d t\|u\|^{m}$ holds for $u \in V$ and $\|u\| \geq M^{\prime \prime}$, where $V$ is an arbitrary subspace of $W_{T}^{1, p}$ and $\operatorname{dim} V<$ $\infty$.

Then problem (1.1) has infinite periodic solutions in $W_{T}^{1, p}$.

Proof. From $\left(B_{5}\right), \varphi$ is an even functional satisfying $\varphi(\theta)=0$. For $u \in \tilde{W}_{T}^{1, p}$,

$$
\int_{0}^{T} F(t, u) d t \geq-\int_{0}^{T} e(t)|u|^{\beta} d t \geq-\|u\|_{\infty}^{\beta} \int_{0}^{T} e(t) d t \geq-T^{\frac{\beta}{q}}\|\dot{u}\|_{L^{p}}^{\beta} \int_{0}^{T} e(t) d t
$$

holds. Then, by (3.1),

$$
\begin{aligned}
\varphi(u) & =\frac{1}{p}\|\dot{u}\|_{L^{p}}^{p}+\int_{0}^{T} F(t, u) d t \geq \frac{1}{p}\|\dot{u}\|_{L^{p}}^{p}-T^{\frac{\beta}{q}}\|\dot{u}\|_{L^{p}}^{\beta} \int_{0}^{T} e(t) d t \\
& \geq \frac{1}{p(T+1)^{p}}\|u\|^{p}-T^{\frac{\beta}{q}} \int_{0}^{T} e(t) d t\|u\|^{\beta}
\end{aligned}
$$

holds. Choose $M^{\prime}>\rho_{1}>0$ small enough such that $\rho_{2}=\frac{1}{p(T+1)^{p}} \rho_{1}^{p}-$ $T^{\frac{\beta}{q}} \int_{0}^{T} e(t) d t \rho_{1}^{\beta}>0$. Then, one has $\varphi(u) \geq \rho_{2}>0$, that is, there exists $\rho_{1}>0, \rho_{2}>0, \varphi(u) \geq \rho_{2}$ holds for $u \in\left\{u \in \tilde{W}_{T}^{1, p}:\|u\|=\rho_{1}\right\}$.

Suppose $\left(A_{2}\right)$ is not satisfied, that is, there exists $V_{1} \subset W_{T}^{1, p}$ which is a subspace with finite dimension such that $\left\{u \in V_{1}: \varphi(u) \geq 0\right\}$ is unbounded. Then, there exist $\left(u_{n}\right)_{n \in \mathbb{N}} \subset V_{1},\left\|u_{n}\right\| \rightarrow \infty$ as $n \rightarrow \infty$, and $\varphi\left(u_{n}\right) \geq 0$. Moreover, we may assume $\left\|u_{n}\right\| \geq M^{\prime \prime}$. Then,

$$
\varphi\left(u_{n}\right)=\frac{1}{p}\left\|\dot{u}_{n}\right\|_{L^{p}}^{p}+\int_{0}^{T} F\left(t, u_{n}\right) d t \leq \frac{1}{p}\left\|\dot{u}_{n}\right\|_{L^{p}}^{p}-\left\|u_{n}\right\|^{m} \int_{0}^{T} l(t) d t
$$




$$
\begin{aligned}
& \leq \frac{1}{p}\left(\int_{0}^{1}\left|u_{n}\right|^{p} d t+\int_{0}^{1}\left|\dot{u}_{n}\right|^{p} d t\right)-\left\|u_{n}\right\|^{m} \int_{0}^{T} l(t) d t \\
& =\frac{1}{p}\left\|u_{n}\right\|^{p}-\left\|u_{n}\right\|^{m} \int_{0}^{T} l(t) d t .
\end{aligned}
$$

Hence, we have $\varlimsup_{n \rightarrow \infty} \varphi\left(u_{n}\right)=-\infty$, which contradicts with $\varphi\left(u_{n}\right) \geq 0$. Then $\left(A_{2}\right)$ is satisfied. Hence, $\varphi$ has infinite critical points in $W_{T}^{1, p}$. Therefore, (1.1) has infinite solutions.

Remark 3.2. From Theorem 2.2, we have the Hamiltonian system (1.1) has infinitely non-trivial solutions.

Similarly, we have the following theorem.

Theorem 3.3. Assume $F(t, u)$ satisfy $(A)$ and $\left(B_{1}\right)-\left(B_{5}\right)$ and the following conditions:

$\left(B_{6}^{\prime}\right)$ there exists $e(t) \in L^{1}\left(0, T ; \mathbb{R}^{+}\right)$satisfying $\frac{1}{p}>T^{\frac{p}{q}} \int_{0}^{T} e(t) d t$ and a constant $M^{\prime}>0$ such that $F(t, u) \geq-e(t)|u|^{p}$ holds for $u \in \tilde{W}_{T}^{1, p}$ and $\|u\| \leq M^{\prime}$

$\left(B_{7}^{\prime}\right)$ there exists $l(t)$ satisfying $\frac{1}{p}<\int_{0}^{T} l(t) d t$ and a constant $M^{\prime \prime}>M^{\prime}$ such that $\int_{0}^{T} F(t, u) d t \leq-\int_{0}^{T} l(t) d t\|u\|^{p}$ holds for $u \in V$ and $\|u\| \geq M^{\prime \prime}$, where $V$ is an arbitrary subspace of $W_{T}^{1, p}$ and $\operatorname{dim} V<\infty$.

Then problem (1.1) has infinite periodic solutions in $W_{T}^{1, p}$.

Example 3.1. Let $T=\frac{1}{3}, \gamma=\frac{11}{2}$, and $F: \mathbb{R} \times \mathbb{R} \rightarrow \mathbb{R}$ as

$$
F(t, x)=\left\{\begin{array}{c}
-2|x|^{2}+|x|-3|x|^{3},|x|<1 \\
-2|x|^{2}-9|x|+9-2|x|^{-\frac{1}{2}},|x| \geq 1 .
\end{array}\right.
$$

Here, $G(x)=-2|x|^{2}$ and

$$
H(t, x)=\left\{\begin{array}{c}
|x|-3|x|^{3},|x|<1 \\
-9|x|+9-2|x|^{-\frac{1}{2}},|x| \geq 1 .
\end{array}\right.
$$

It is obvious that $\alpha=0, F(t, 0)=0, F(t,-x)=F(t, x)$. Moreover, $F(t, x) \geq$ $-4|x|^{2}$ if $|x|<1$ and there exists $M^{\prime \prime}>1$ such that $-9|x|+9-2|x|^{-\frac{1}{2}}<0$ if $|x| \geq M^{\prime \prime}$, so, $\int_{0}^{T} F(t, x) d t \leq-\frac{2}{3}|x|^{2}$. Hence, $F(t, x)$ satisfies all conditions of Theorem 3.3.

\section{References}

[1] R. A. Adams, Sobolev Space, Academic Press. New York, 1975.

[2] J. Mawhin and M. Willem, Critical Point Theory and Hamiltonian Systems, Applied Mathematical Sciences, 74. Springer-Verlag, New York, 1989.

[3] P. H. Rabinowitz, Minimax Methods in Critical Point Theory with Applications to Differential Equations, CBMS Regional Conference Series in Mathematics, 65. Published for the Conference Board of the Mathematical Sciences, Washington, DC; by the American Mathematical Society, Providence, RI, 1986. 
[4] C. L. Tang, Periodic solutions of non-autonomous second order systems with $\gamma$ quasisubadditive potential, J. Math. Anal. Appl. 189 (1995), no. 3, 671-675.

[5] C. L. Tang and X. P. Wu, Periodic solutions for second order systems with not uniformly coercive potential, J. Math. Anal. Appl. 259 (2001), no. 2, 386-397.

[6] X. P. Wu and C. L. Tang, Periodic solutions of a class of non-autonomous second-order systems, J. Math. Anal. Appl. 236 (1999), no. 2, 227-235.

[7] Y. W. Ye and C. L. Tang, Periodic solutions for some nonautonomous second order Hamiltonian systems, J. Math. Anal. Appl. 344 (2008), no. 1, 462-471.

Li ZHANG

Department of Mathematics

Beijing Institute of Technology

Beijing, 100081, P. R. China

E-mail address: amy_zhangli@sina.com

Weigao Ge

Department of Mathematics

Beijing Institute of Technology

Beijing, 100081, P. R. China

E-mail address: gew@bit.edu.cn 